\section{G320(P) POST-MALARIA NEUROLOGICAL SYNDROME: THE FIRST IRISH PAEDIATRIC CASE}

${ }^{1} S$ Almuntaser, ${ }^{1}$ A Costello, ${ }^{1}$ B Lynch, ${ }^{2}$ J Leonard, ${ }^{3} \mathrm{P}$ Gavin. ${ }^{1}$ Neurology, Temple Street Children's University Hospital, Dublin, Ireland; ${ }^{2}$ General Paediatric, Temple Street Children's University Hospital, Dublin, Ireland; ${ }^{3}$ Infectious Disease, Temple Street Children's University Hospital, Dublin, Ireland

\subsection{6/archdischild-2018-rcpch.310}

Aims Post-malaria neurological syndrome (PMNS) is described as a rare post-infectious encephalopathy occurring within two months of resolved malaria infection and with an aparasitaemia. PMNS encompasses three separate neurological syndromes: A delayed cerebellar syndrome, an acute demyelinating polyneuropathy (GBS) and an acute disseminated encephalopathy (ADEMs).

Here, we report the first Irish paediatric case of falciparum PMNS, in a patient of African origin, born and living in Ireland.

A 15 year old boy presented with a 3 day history of progressive encephalopathy, features of raised ICP and seizures on a background of falciparum malaria treated six weeks previously. PMNS was diagnosed after further investigations and an aparasitaemia. He was sedated and intubated for 2 days and commenced on antimicrobials, antimalarial and steroids. His investigations results as following: MRI brain: Cerebral oedema and optic neuritis, EEG: Severe encephalopathy. Serial thick and thin films: No malaria parasites Falciparum Protein Antigen (RDT): Positive (Can remain positive for 6 weeks after malaria). CSF Studies: Protein $1383 \mathrm{~g} / \mathrm{dl}, 16 \mathrm{WBCs} / \mathrm{dl}$, 100\% mononuclear cells). PCRs: negative for HSV, Adenovirus, Coxsackie, EBV, CMV, Meningococcus, Pneumococcus.

By day 6 of admission he had made a full recovery with no neurological deficits.

Methods Using Google and PubMed, we searched for relevant case reports and journal articles describing neurological syndromes occurring post infection with falciparum malaria in the paediatric population.

Results Whilst the prevalence of PMNS (plasmodium falciparum) is $0.12 \%$ in adults, the prevalence in children remains unknown. In 1996, a Vietnamese study conducted over 4 years reported 23 patients with PMNS following full recovery from falciparum malaria. Of these, only 3 were children. A 2015 case report describes a further two children with falciparum PMNS. There have been no further paediatric cases reported to date worldwide.

Conclusion In conclusion, PMNS is an increasingly recognised, but rare complication of malaria that must be differentiated from relapsing or recurrent malaria, and post-infectious neurological syndromes, e.g. ADEM. In particularly severe cases, steroids have been given as an adjunctive therapy to speed recovery however PMNS is a self-limiting condition that resolves within 2-14 days and requires no specific treatment.

\section{G321(P) SPONTANEOUS REMISSION OF EPILEPSY IN A CASE OF MESIAL TEMPORAL SCLEROSIS}

R Manuel, L Yates, S Philip. Paediatric Neurology, Birmingham Children's Hospital NHS Trust, Birmingham, UK

10.1136/archdischild-2018-rcpch.311

Background Mesial temporal sclerosis (MTS) is commonly referred to as hippocampal sclerosis (HS), is the most common association with intractable temporal lobe epilepsy. The typical MRI features of hippocampal sclerosis, are unilateral volume loss and increased signal intensity on T2-weighted images. There is no sex or side preference and a proportion of cases are bilateral. Macroscopically the hippocampus is firm and shrunken. Microscopic findings include a characteristic pattern of neuronal loss and reactive gliosis that varies in severity from case to case. Mesial temporal sclerosis is an uncommon finding in children, but when it occurs, it is always associated with epilepsy. Mesial temporal sclerosis is the most frequent cause of drug-resistant temporal lobe epilepsy but has a satisfactory response to surgery, and is considered infrequent in children.

Patient Characteristics: 13 year old girl, she was born at 41 week of gestation with an uneventful antenatal history. She was ventilated at birth and also treated for streptococcal meningitis. She developed seizures during this treatment period. She didn't require antiepileptic at discharge. No developmental concerns. She presented with focal seizures at the age of 3 years. Had further seizures, about one or two a year, up to the age of 6 years. Eye deviation to right side, abnormal mouthing movements, disoriented, confused, vacant, lip smacking lasting for $10 \mathrm{~min}$ followed by headache and vomiting with post ictal phase for two hours. She remained seizure free for the past 6 years. She was not commenced on any medication.

Investigations EEG at the age of 5 years shown clustered spike epileptiform discharges focal in the central, temporal and occipital area.

MRI scan - MRI brain scan showed dilatation of the left temporal horn with left mesial temporal sclerosis.

Conclusion This is a case of mesial temporal sclerosis with spontaneous remission of epilepsy. It is extremely unusual for mesial temporal sclerosis to be spontaneously seizure free. Pathophysiological mechanism of epilepsy in mesial temporal sclerosis is not fully understood. Further imaging and pathological studies comparing similar cases against the usual MTS with drug resistant epilepsy might lead to find clinically useful indicators.

\section{G322(P) NOT BELL'S PALSY ANYMORE? LYME DISEASE (LD) UNTIL PROVEN OTHERWISE}

${ }^{1} \mathrm{~S}$ Penrose, ${ }^{1} \mathrm{H}$ Walsh, ${ }^{1} \mathrm{Y}$ Kumar. 'Paediatric Department, Royal Cornwall Hospital, Truro, UK; ${ }^{2}$ Paediatric Speciality Trainee, Royal Cornwall Hospital, Truro, UK; ${ }^{3}$ Paediatric Consultant, Royal Cornwall Hospital, Truro, UK

\subsection{6/archdischild-2018-rcpch.312}

Aims Lyme Borreliosis (LD) is becoming increasingly prevalent across parts of the UK. Recent evidence suggests that LD is the commonest cause of lower motor neuron type facial palsy (LMN FP) in children and adults in the USA. Historically, idiopathic LMN FP, termed Bell's palsy, was given as the commonest cause in the UK, we discuss whether current evidence suggests otherwise.

Methods We report 2 cases of LMN FP seen over an evening shift, which were subsequently serologically confirmed cases of LD. We also reviewed current literature and surveillance data. Results LD is an infectious disease caused by the spirochaete Borrelia burgdorferi. It is the most common tick-borne infectious disease in the UK and is becoming increasingly prevalent in certain areas, affecting around 9.8/100 000, a figure that continues to rise. The presenting features are often non- 